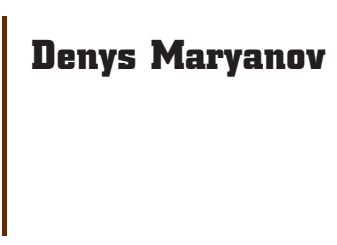

\title{
DEVELOPMENT OF A METHOD FOR MAINTAINING THE PERFORMANCE OF DRILLING FLUIDS DURING TRANSPORTATION BY PLATFORM SUPPLY VESSEL
}

The object of research is the process of transportation of drilling fluid used for lubrication and cooling of drilling equipment of offshore oil production platforms. The subject of the study is the stratification of the density of the drilling fluid along the height of the cargo tank in which it is transported. The technology of transportation of drilling fluid on the Platform Supply Vessel is considered. A problematic point in ensuring this process is that during the transportation of drilling fluids, due to the action of gravitational forces on organic and inorganic compounds in their volume, there is a latent change in their dispersion over the volume of the fluid. This leads to the stratification and stratification of the fluid density along the height, as well as to the formation of sediments at the bottom of the cargo tanks, in which the drilling fluid is transported. The study is aimed at developing a technology that maintains a constant value of the density of the drilling fluid along the depth of the tank in which it is transported. The studies were carried out in the vessel system for transporting drilling fluid of a specialized marine vessel of the Platform Supply Vessel type with a deadweight of 5850 tons. It is experimentally established that for a transportation time of 6-36 hours, the density stratification of the drilling fluid is 3.04-32.04\%. As a method that ensures the minimum stratification of the density of the drilling fluid during its transportation, it is proposed to use an additional $X$-shaped circulation of the drilling fluid in the volume of adjacent cargo tanks. Studies have confirmed that the density stratification over a time period of 6-36 hours decreases to a range of $2.30-9.01 \%$. The complex use of additional X-shaped circulation and simultaneous air supply to the bottom of the cargo tank provides a density stratification value of 0.73-2.93\%. The proposed technology was tested on a specialized seagoing vessel of the Platform Supply Vessel type with a deadweight of 5850 tons and can be used on offshore vessels that ensure the operation of offshore oil production platforms.

Keywords: Platform Supply Vessel, drilling fluid, transportation system, density stratification.

\section{Introduction}

Every year all countries with oil reserves increase its production. This is due to the constant growth in the consumption of thermal, mechanical and electrical energy, the source of which is liquid fuel. Oil fields are not only onshore. There are offshore fields, the main of which are the Persian, Venezuelan and Guinean Gulfs, as well as the North Sea. The development, production and transportation of oil in offshore areas (in comparison with continental sources) is characterized by an increased complexity of the technological process, as well as the use of special engineering structures (drilling platforms) and special purpose marine vessels - Platform Supply Vessel (PSV). These vessels provide delivery of water, fuel, oil, food, necessary equipment and special technical fluids (drilling fluids) from the shore to the offshore drilling platform [1].
The functional properties of drilling fluids include the provision of lubrication, cooling and anti-corrosion action on the drilling tool [2, 3]. Drilling fluid is a complex multicomponent dispersed system, the dispersed medium of which is a lubricant of petroleum origin, and various organic and inorganic compounds are used as the dispersed phase, the specific gravity of which exceeds the specific gravity of the lubricant $[4,5]$. Depending on the type and amount of these compounds, drilling fluids have different physical (primarily density and viscosity) and rheological (primarily shear resistance and fluidity) characteristics [6, 7]. In the process of transportation of drilling fluids, due to the action of gravitational forces on organic and inorganic compounds, a latent change in their dispersion over the volume of the fluid occurs. This leads to stratification of the drilling fluid and stratification of its density along the height, as well as to the formation of sediments at the 
bottom of the cargo tanks in which it is transported. In this case, situations are possible when the amount of high-density sediment does not allow for pumping the drilling fluid through the pipelines of the system and pumping it out (transferring) to the oil production platform. Removal of sediment manually or mechanically belongs to the category of work unusual for the vessel's crew. Their implementation is associated with an increase in the sailing time of the vessel, leads to losses of thermal [8] and mechanical energy [9], therefore, increases financial costs and reduces the economic performance of PSV.

Thus, maintaining the operational characteristics of drilling fluids during their transportation by PSV-class sea vessels is an urgent applied scientific and technical problem.

\section{The object of research and its technological audit}

The object of research is the process of transportation of drilling fluid used for lubrication and cooling of drilling equipment of offshore oil production platforms. The subject of research is the stratification of the density of the drilling fluid along the height of the cargo tank in which it is transported.

Maintaining the rheological characteristics (viscosity, dispersity, density) of drilling fluids ensures reliable operation of the equipment and the continuity of the process of production and transportation of hydrocarbons. In most cases, these issues are considered in relation to continental fields, for conditions that do not take into account the specifics of the transportation and transfer of drilling fluids to offshore or ocean drilling platforms [10].

The vector of scientific research aimed at optimizing the operational characteristics of working substances used in vessel power plants is aimed at studying the properties of marine heavy fuels and motor oils [4,6]. The issues of ensuring the functional properties and performance of technical fluids, such as drilling fluids, are practically not studied in relation to the marine industry. The rules for their transportation have no confirmed practical recommendations. Latent deterioration of their rheological characteristics cannot always be determined, evaluated and eliminated by the vessel's crew.

\section{The aim and objectives of research}

The aim of research is to develop a technology that maintains a constant value of the density of the drilling fluid along the depth of the tank in which it is transported. This will provide:

- maintaining the operational characteristics of the drilling fluid;

- minimization of sediment formation of heavy components with which the drilling fluid is doped;

- reduction of energy costs for the process of pumping drilling fluid from the PSV to the drilling platform;

- maintenance of the technical condition of the vessel drilling fluid transportation system.

This aim can be achieved by solving the following objectives:

1. Monitor the density of the drilling fluid along the height of the cargo tank.

2. Consider ways to modernize the vessel's drilling fluid storage and transportation system.
3. Determine the parameters for ensuring forced circulation of the drilling fluid.

4. Propose parameters for maintaining the rheological characteristics of the drilling fluid during its transportation.

\section{Research of existing solutions to the problem}

To maintain and restore the rheological characteristics (in particular, viscosity and density) of technical fluids, their hydrodynamic, ultrasonic and chemical treatment are used $[11,12]$.

The hydrodynamic effect on the fluid flow is carried out by creating an increased pressure and increasing the speed of its movement $[13,14]$. In this case, due to the fragmentation of the elements of the dispersed phase, their dispersion and uniformity of the entire volume of the liquid increase [15, 16].

During ultrasonic treatment in a fluid flow, there is a pulse increase and decrease in pressure in the local volumes of the fluid. This, as well as the provision of turbulent motion, leads to an increase in the homogeneity of the liquid and the prevention of sediment of heavy components that make up its composition [17, 18].

Maintaining and restoring the rheological characteristics of technical fluids is also possible by chemical treatment. In this case, additional reagents are introduced into the volume of the liquid, which, due to intermolecular interactions, ensure the maintenance of the homogeneity of the liquid at the required level during the periods of storage and transportation $[19,20]$.

The above methods for solving the problem of maintaining the rheological characteristics of technical fluids are used for fuels and oils used in power plants of vessels of sea and river transport. An optimal solution to the important problem of maintaining the physical and rheological characteristics of drilling fluids during their transportation by vessels of the PSV class has not yet been found.

\section{Methods of research}

To determine the technology that ensures the maintenance of a constant value of the density of the drilling fluid along the depth of the tank in which it is transported, experimental studies were carried out on the PSV sea vessel with a deadweight of 5850 tons. Vessels of the PSV type belong to the medium-speed class (their maximum speed, as a rule, does not exceed 12-13 knots), therefore the duration of their passage (and, accordingly, the time of transportation of the drilling fluid) from the port to the oil platform is $2-5$ days. In addition, there may be cases where some operational and technological reasons necessitate drifting or parking near drilling platforms. This time interval contributes to the gradual deposition of heavy components, which are alloyed in the fluids, and increases the stratification of the density of the drilling fluid along the depth of the cargo tank in which it is located $[1,20]$.

The fluid was transported in six tanks of the same type, located symmetrically on the left and right sides. The system provides for technological operations for taking on board and delivering drilling fluid to the oil platform, which are provided by cargo pumps. In order to minimize the process of stratification of the density of the drilling fluid, to prevent its stratification and the formation 
of sediment from heavy components, the vessel's drilling fluid transportation system was modernized by:

- installation of additional pipelines that ensured X-

shaped circulation of the fluid in the tanks;

- installation of aerodynamic inserts in the lower part

of the cargo tank, through which air was supplied.

The changes made to the system are shown in Fig. 1.
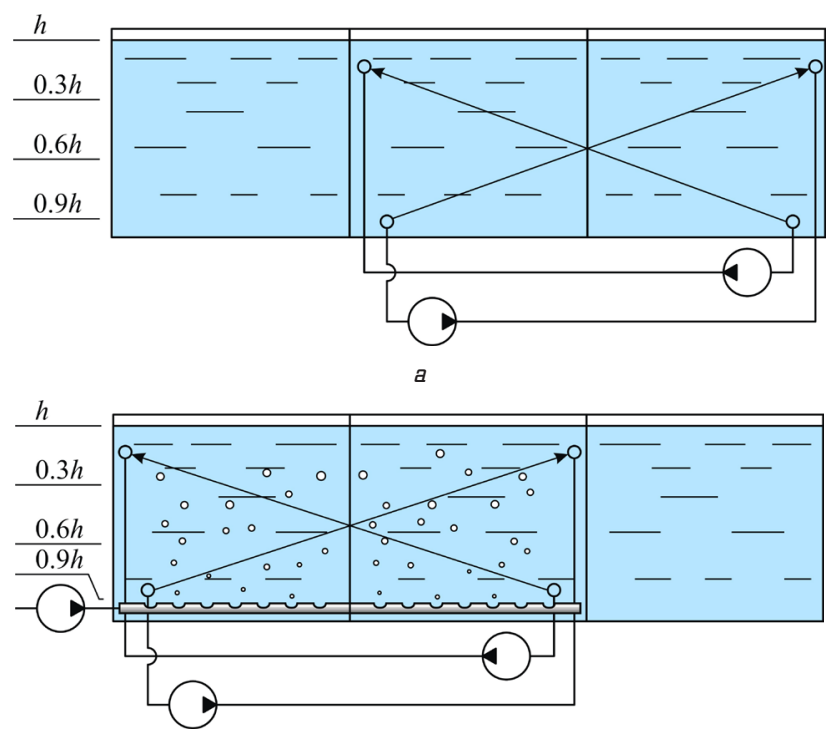

Fig. 1. Schematic diagram of the inclusion of additional units in the vessel drilling fluid transportation system: $a$ - ensuring the X-shaped circulation of the drilling fluid in the tanks; $b$ - ensuring the X-shaped circulation of the drilling fluid in the tanks with simultaneous air supply

The changes made to the system were carried out by the vessel's crew and agreed with the technical department of the shipping company. The complexity of the work performed did not exceed 10 hours. The work was carried out during the ballast passage of the vessel.

\section{Research results}

Density was taken as the control parameter by which the dispersed state of the drilling fluid was estimated. Its determination was carried out by an Anton Paar DMA35 Tag\&Log electronic hydrometer from LEMIS Baltic (Latvia-Germany), which allows measurements in the range of $650-1630 \mathrm{~kg} / \mathrm{m}^{3}$ with an accuracy of $\pm 1 \mathrm{~kg} / \mathrm{m}^{3}$ while simultaneously monitoring the temperature of the measured samples [21, 22].

Samples for determining the density of the drilling fluid were taken at various points of the tank, corresponding to 30,60 and $90 \%$ of its depth. For the experiment, the drilling fluid was transported under the following conditions:

1) two tanks without changing the design of the system;

2) two tanks, in which the X-shaped circulation of the drilling fluid was additionally provided - Fig. 1, $a$;

3) two tanks, in which X-shaped circulation of the drilling fluid and forced air supply were additionally provided - Fig. 1, $b$.

The time of the PSV's ocean passage from the port to the drilling platform was 44 hours, which made it possible to sample the drilling fluid for all experimental conditions within 36 hours with an interval between density measurements of 6 hours. The research results are shown in Table 1.
Table 1

Change in the density of the drilling fluid $\left(\rho, \mathrm{kg} / \mathrm{m}^{3}\right)$ along the depth of the cargo tank, depending on the time and method of its transportation

\begin{tabular}{|c|c|c|c|c|c|c|c|}
\hline \multicolumn{8}{|c|}{ Transport without changing the system design } \\
\hline \multirow{2}{*}{$\begin{array}{c}\text { Measurement } \\
\text { level }\end{array}$} & \multicolumn{7}{|c|}{ Transportation time, hours } \\
\hline & 0 & 6 & 12 & 18 & 24 & 30 & 36 \\
\hline on a surface & 1232 & 1218 & 1205 & 1188 & 1173 & 1135 & 1105 \\
\hline $0.3 h$ & 1232 & 1230 & 1222 & 1205 & 1189 & 1178 & 1152 \\
\hline $0.6 h$ & 1233 & 1248 & 1265 & 1285 & 1301 & 1345 & 1406 \\
\hline $0.9 h$ & 1235 & 1255 & 1282 & 1312 & 1342 & 1385 & 1463 \\
\hline \multicolumn{8}{|c|}{ Transportation with additional X-shaped circulation } \\
\hline on a surface & 1272 & 1235 & 1175 & 1122 & 1068 & 1028 & 1002 \\
\hline $0.3 h$ & 1232 & 1220 & 1215 & 1202 & 1182 & 1179 & 1176 \\
\hline $0.6 h$ & 1233 & 1226 & 1222 & 1208 & 1196 & 1194 & 1185 \\
\hline $0.9 h$ & 1233 & 1242 & 1246 & 1246 & 1249 & 1258 & 1262 \\
\hline \multicolumn{8}{|c|}{$\begin{array}{c}\text { Transportation with additional X-shaped circulation and air supply to the } \\
\text { volume of the cargo tank }\end{array}$} \\
\hline on a surface & 1233 & 1232 & 1231 & 1228 & 1227 & 1226 & 1226 \\
\hline $0.3 h$ & 1233 & 1232 & 1232 & 1232 & 1231 & 1231 & 1230 \\
\hline $0.6 h$ & 1235 & 1236 & 1242 & 1245 & 1248 & 1251 & 1253 \\
\hline $0.9 h$ & 1236 & 1241 & 1248 & 1251 & 1258 & 1262 & 1263 \\
\hline
\end{tabular}

Graphical dependencies that show the change in the density of the drilling fluid over time for different conditions of its transportation are shown in Fig. 2.

The results, which are shown in Table 1 and Fig. 2 indicate that the decrease/increase in the density of the drilling fluid occurs exponentially. The maximum increase in density $\rho_{\max }^{+}$(and the corresponding weighting of the drilling fluid) corresponds to the lower part of the cargo tank (90\% depth). The maximum decrease in density $\rho_{\max }^{-}$corresponds to the layer of drilling fluid that is on the surface of the cargo tank.

The highest values $\rho_{\max }^{+}$and $\rho_{\max }^{-}$corresponds to the transportation of the drilling fluid without changing the design of the system. In this case, the stratification of the density of the drilling fluid along the depth of the cargo tank:

$$
\Delta \rho=\frac{\rho_{h}-\rho_{0.9 h}}{\rho_{h}} \cdot 100 \%,
$$

where $\rho_{h}$ - density of the drilling fluid on the surface of the cargo tank, $\mathrm{kg} / \mathrm{m}^{3} ; \rho_{0.9 h}$ - density of the drilling fluid at a depth of $90 \%, \mathrm{~kg} / \mathrm{m}^{3}$ (for a transportation time of $6-36 \mathrm{~h}$, it is $\Delta \rho=3.04-32.04 \%$ ).

In the case of modernization of the system, the density stratification was:

- with additional X-shaped circulation $-\Delta \rho=2.30-9.01 \%$;

- with additional X-shaped circulation and simultaneous

air supply $-\Delta \rho=0.73-2.93 \%$.

The effectiveness of the proposed methods can be estimated from Fig. 2 as the area bounded by the exponents of the maximum increase $\rho_{\max }^{+}$and maximum decrease $\rho_{\max }^{-}$ in density over a selected period of time.

These areas $\left(S_{0}^{6}, S_{6}^{12}, S_{12}^{18}, S_{18}^{24}, S_{24}^{30}, S_{30}^{36}\right)$ can be calculated as the areas of the corresponding trapezoids [4, 23]. The values of the trapezoid areas (which characterize the density stratification of the drilling fluid along the depth 
of the cargo tank and are a criterion for assessing the density stratification) are given in Table 2. The area of these trapeziums has the dimension $\left(\mathrm{kg} \cdot \mathrm{h} / \mathrm{m}^{3}\right)$, however, further (in the text and in Table 2) only numerical values are indicated. The increase in area corresponds to an increase in density stratification along the depth of the cargo tank and the formation of sludge of heavy components of the drilling fluid.

The results of Table 2 are summarized in the form of a nomogram shown in Fig. 3.
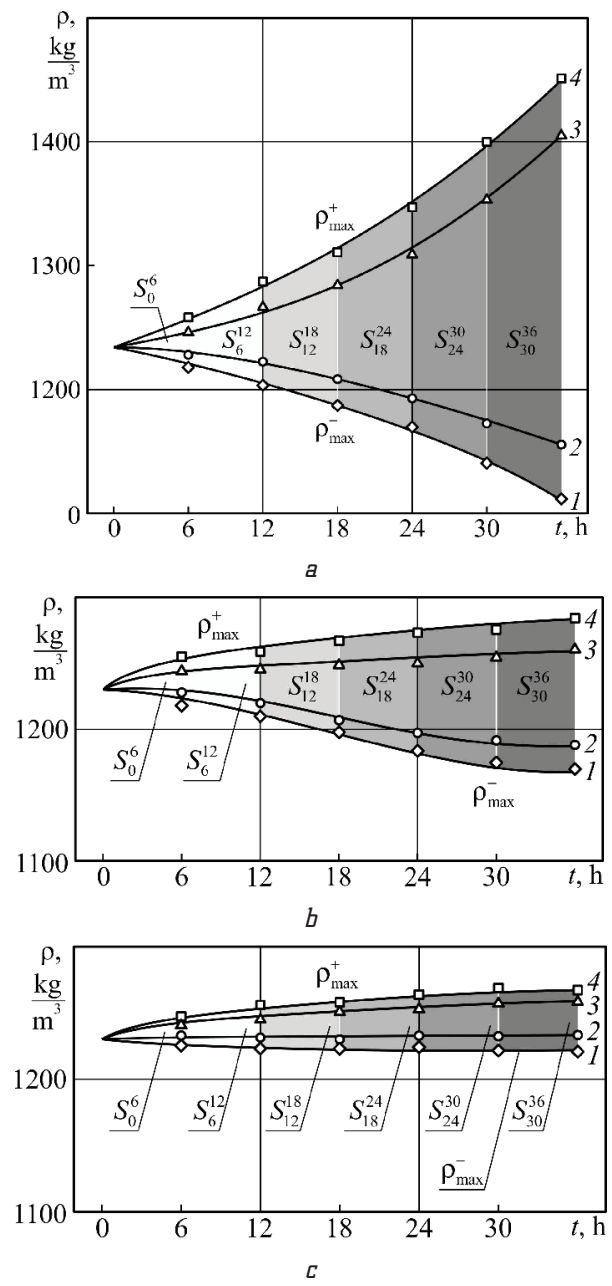

Fig. 2. Changes in the density of the drilling fluid under different conditions of its transportation: $a$ - transportation without changing the design of the system; $b$ - with additional X-shaped circulation; $c$ - with additional X-shaped circulation and simultaneous air supply; 1 - density

of the drilling fluid on the surface of the tank; 2, 3, 4 - at a level

corresponding to $30 \%, 60 \%$, $90 \%$ of the depth of the tank

Table 2

Criterion for assessing the stratification of the density of the drilling fluid along the depth of the cargo tank, depending on the time and method of its transportation

\begin{tabular}{|c|c|c|c|c|c|c|}
\hline \multirow{2}{*}{$\begin{array}{c}\text { Transportation } \\
\text { method }\end{array}$} & \multicolumn{6}{|c|}{ Transportation time, hours } \\
\cline { 2 - 7 } & 6 & 12 & 18 & 24 & 30 & 36 \\
\hline 1 & 120 & 342 & 603 & 879 & 1257 & 1824 \\
\hline 2 & 96 & 216 & 312 & 438 & 537 & 597 \\
\hline 3 & 36 & 78 & 120 & 162 & 201 & 219 \\
\hline
\end{tabular}

Note: 1 - without changing the design of the system; 2 - transportation with additional X-shaped circulation; 3 - transportation with additional $\mathrm{X}$-shaped circulation and air supply to the volume of the cargo tank

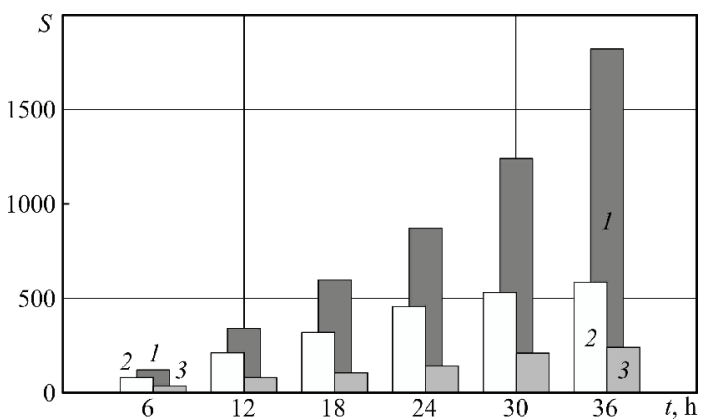

Fig. 3. Criterion for assessing the stratification of the density of the drilling fluid depending on time for different transportation conditions:

1 - without changing the design of the system; 2 - additional X-shaped circulation; 3 - additional X-shaped circulation and forced air supply to the bottom of the cargo tank

The nomograms shown in Fig. 3 confirm the effectiveness of the proposed methods for maintaining the density of the drilling fluid. None of the options can completely prevent the process of settling of heavy components that make up the drilling fluid. Moreover, their use contributes to a 3.6-10.9-fold decrease in density stratification.

\section{SWOT analysis of research results}

Strengths. The strengths of this study are that the proposed method provides the minimum values of the density stratification of the drilling fluid along the depth of the tank in which it is transported. It also prevents the drilling fluid from stratifying and the formation of sediment of heavy components included in its composition. Maintaining the required density of the drilling fluid minimizes the time it takes to pump it from the vessel to the drilling platform and reduces energy costs for this operation.

Weaknesses. The weaknesses of this study are related to the fact that to use the proposed method, it is necessary to re-equip the vessel drilling fluid transportation system. Such works can be performed only by agreement with the vessel owner, and in some cases with the Register, which controls the operation of the sea vessel and its power plant.

Opportunities. Application of the proposed technology for maintaining the rheological characteristics of technical fluids is possible in various facilities of the technological complex to ensure oil production (for example, onshore bases providing production and temporary storage of drilling fluid, as well as in transport organizations carrying out the transportation of drilling fluid from onshore bases to the PSV vessel).

Threats. The option of maintaining the rheological characteristics of technical fluids proposed in this work is of an applied nature and is based on practical research. For the analytical calculation of the optimal processing time for the drilling fluid, which ensures the minimum stratification of its density with the simultaneous minimum energy consumption for the processing process, it is necessary to develop a mathematical model and perform mathematical modeling [24, 25].

\section{Conclusions}

1. It is found that to monitor the density of the drilling fluid, it is necessary to perform measurements at levels cor- 
responding to $30 \%, 60 \%, 90 \%$ of the depth of the tank. It has been experimentally established that for a transportation time of $6-36 \mathrm{~h}$, the density stratification of the drilling fluid is $3.04-32.04 \%$.

Thus, heavy components of the drilling fluid, settling to the bottom of the tank, subsequently complicate the process of pumping it out. This can lead to breakdown or failure of cargo pumps, inability to pump drilling fluid onto the drilling platform and further disruption of the oil production process.

2. It is shown that in order to reduce the stratification of the drilling fluid density, it is advisable to modernize the vessel's storage and transportation system. The paper proposes the following upgrade options:

a) the use of additional X-shaped circulation of the drilling fluid in the volume of adjacent cargo tanks (while pumping the drilling fluid between the lower and upper parts of the tanks);

b) the complex use of additional X-shaped circulation and simultaneous air supply to the bottom of the cargo tanks.

In this case (in the case of transportation of the drilling fluid from the shore to the drilling platform in $6-36 \mathrm{~h}$ ), the density stratification is:

- option $a-2.30-9.01 \%$;

- option $b-0.73-2.93 \%$.

The decrease in density stratification for these transportation options is explained by the artificial creation of a laminar flow of the drilling fluid (due to its forced circulation between adjacent cargo tanks). As well as its local turbulent currents (due to additional air supply to the lower part of the cargo tanks). The cross movement of the drilling fluid (which is carried out both under the action of gravitational forces and with the help of additionally installed circulation pumps) and air can contribute to the occurrence of the cavitation phenomenon. This leads to a forceful effect on organic and inorganic compounds in the volume of the drilling fluid, which prevents their sedimentation and maintains them in fluid.

3. It is determined that the modernized system, which provides the minimum level of density stratification of the drilling fluid during its transportation on PSV-type vessels, includes a mobile circulation pump and an air compressor, pipelines and fittings. It can also be installed on a specialized vessel in accordance with the technological scheme directly by the vessel's crew. The power required to ensure the functioning of such a system does not significantly affect the energy performance of the vessel power plant, which is characterized by increased values and a large safety factor for specialized vessels that ensure the operation of drilling platforms.

4. The following technological solutions are proposed: additional $\mathrm{X}$-shaped circulation of the drilling fluid, as well as X-shaped circulation with simultaneous air supply to the bottom of the cargo tank. These solutions provide a 1.3-3.6 fold (in the first case) and $4.2-10.9$ fold (in the second case) decrease in density stratification - the most important rheological characteristic of the drilling fluid. This prevents the formation of sediment of heavy components of the drilling fluid, reduces the energy load on the vessel's power plant during pumping of the drilling fluid to the drilling platform. It also reduces the pumping time of the drilling fluid and helps to ensure the technological process of oil production.

\section{References}

1. Karianskyi, S. A., Maryanov, D. M. (2020). Features of transportation of high-density technical liquids by marine specialized vessels. Scientific research of the SCO countries: synergy and integration. Beijing, 2, 150-153.

2. Lipin, A. A., Kharlamov, Y. P., Timonin, V. V. (2013). Circulation system of a pneumatic drill with central drilling mud removal. Journal of Mining Science, 49 (2), 248-253. doi: http://doi.org/ $10.1134 / \mathrm{s} 1062739149020068$

3. Liang, Y., Ju, X., Li, A., Li, C., Dai, Z., Ma, L. (2020). The Process of High-Data-Rate Mud Pulse Signal in Logging While Drilling System. Mathematical Problems in Engineering, 2020, 1-11. doi: http://doi.org/10.1155/2020/3207087

4. Zablotsky, Y. V., Sagin, S. V. (2016). Enhancing Fuel Efficiency and Environmental Specifications of a Marine Diesel When using Fuel Additives. Indian Journal of Science and Technology, 9 (46), 353-362. doi: http://doi.org/10.17485/ijst/2016/ v9i46/107516

5. Lahoida, A., Boryn, V., Sementsov, G., Sheketa, V. (2020). Development of an automated system of control over a drilling mud pressure at the inlet to a well. Eastern-European Journal of Enterprise Technologies, 4 (2 (106)), 82-94. doi: http://doi.org/ 10.15587/1729-4061.2020.209844

6. Sagin, S. V., Semenov, O. V. (2016). Motor Oil Viscosity Stratification in Friction Units of Marine Diesel Motors. American Journal of Applied Sciences, 13 (2), 200-208. doi: http://doi.org/ 10.3844/ajassp.2016.200.208

7. Sagin, S. V., Solodovnikov, V. G. (2017). Estimation of Operational Properties of Lubricant Coolant Liquids by Optical Methods. International Journal of Applied Engineering Research, 12 (19), 8380-8391.

8. Budashko, V., Obniavko, T., Onishchenko, O., Dovidenko, Y., Ungarov, D. (2020). Main Problems of Creating Energy-efficient Positioning Systems for Multipurpose Sea Vessels. 2020 IEEE 6th International Conference on Methods and Systems of Navigation and Motion Control (MSNMC), 106-109. doi: http:// doi.org/10.1109/msnmc50359.2020.9255514

9. Kuropyatnyk, O. A., Sagin, S. V. (2019). Exhaust Gas Recirculation as a Major Technique Designed to Reduce NOx Emissions from Marine Diesel Engines. Naše More, 66 (1), 1-9. doi: http://doi.org/10.17818/nm/2019/1.1

10. He, J. F., Zhang, P. Y., Yin, Q. L., Yin, K., Liu, H. P. (2015) Study of drilling muds on the anti-erosion property of a fluidic amplifier in directional drilling. Frattura Ed Integrità Strutturale, 9 (34), 564-573. doi: http://doi.org/10.3221/igf-esis.34.62

11. Gnap, J., Senko, Š., Marienka, P. (2020). Time Availability of the Public Terminal of Intermodal Transport Žilina with a Selected European Maritime Port. Naše More, 67 (3), 217-225. doi: http://doi.org/10.17818/nm/2020/3.5

12. Kisel', A. G., Purtov, E. D., Vyborov, S. S., Belan, D. Yu., Grechishnikov, V. A. (2019). Studying the function of the lifespan of carbide tools during turning of workpieces made of alloy $12 \mathrm{H} 18 \mathrm{~N} 10 \mathrm{~T}$ from the cooling properties of the cutting fluids. Journal of Physics: Conference Series, 1260, 062011. doi: http://doi.org/10.1088/1742-6596/1260/6/062011

13. Kondratenko, Y. P., Topalov, A. M., Kozlov, O. V. (2019). Simulation of the Initial Stability of the Floating Dock for the List and Trim Stabilization Tasks. Problems of the Regional Energetics, 1-2 (41), 12-24. doi: http://doi.org/10.5281/ zenodo.3239200

14. Javadian, S., Sadrpoor, S. M. (2020). Demulsification of water in oil emulsion by surface modified $\mathrm{SiO}_{2}$ nanoparticle. Journal of Petroleum Science and Engineering, 184, 106547. doi: http:// doi.org/10.1016/j.petrol.2019.106547

15. Sagin, S. V., Solodovnikov, V. G. (2015). Cavitation Treatment of High-Viscosity Marine Fuels for Medium-Speed Diesel Engines. Modern Applied Science, 9 (5), 269-278. doi: http:// doi.org/10.5539/mas.v9n5p269

16. Shettigar, R. R., Misra, N. M., Patel, K. (2018). CTAB grafted PAM gel and its application in drilling fluid. Journal of Petroleum Science and Engineering, 160, 129-135. doi: http:// doi.org/10.1016/j.petrol.2017.10.040 
17. Shahbazov, E. (2015). Development and application of nanodrilling muds. Scientific Israel-Technological Advantages, 17 (2), 160-171.

18. Sagin, S. V., Kuropyatnyk, O. A. (2018). The Use of Exhaust Gas Recirculation for Ensuring the Environmental Performance of Marine Diesel Engines. Naše More, 65 (2), 78-86. doi: http://doi.org/10.17818/nm/2018/2.3

19. Petcovic, M., Zubcic, M., Krcum, M., Pavic, I. (2021) Wind Assisted Ship Propulsion Technologies - Can they Help in Emissions Reduction? Naše more, 68 (2), 102-109. doi: http:// doi.org/10.17818/nm/2021/2.6

20. Mahmoudpour, M., Pourafshary, P. (2021). Investigation of the effect of engineered water/nanofluid hybrid injection on enhanced oil recovery mechanisms in carbonate reservoirs. Journal of Petroleum Science and Engineering, 196, 107662. doi: http://doi.org/10.1016/j.petrol.2020.107662

21. Sagin, S. V., Semenov, O. V. (2016). Marine Slow-Speed Diesel Engine Diagnosis with View to Cylinder Oil Specification. American Journal of Applied Sciences, 13 (5), 618-627. doi: http://doi.org/10.3844/ajassp.2016.618.627

22. Popovskii, Yu. M., Sagin, S. V., Khanmamedov, S. A., Grebenyuk, M. N., Teregerya, V. V. (1996). Designing, calculation, testing and reliability of machines: influence of anisotropic fluids on the operation of frictional components. Russian Engineering Research, 16 (9), 1-7.

23. Li, X., Zhang, J., Tang, X., Mao, G., Wang, P. (2020). Study on wellbore temperature of riserless mud recovery system by CFD approach and numerical calculation. Petroleum, 6 (2), 163-169. doi: http://doi.org/10.1016/j.petlm.2019.06.006

24. Likhanov, V. A., Lopatin, O. P. (2020). Dynamics of soot formation and burnout in a gas diesel cylinder. IOP Conference Series: Materials Science and Engineering, 862, 062033 doi: http://doi.org/10.1088/1757-899x/862/6/062033

25. Wanderley Neto, A. O., da Silva, V. L., Rodrigues, D. V., Ribeiro, L. S., Nunes da Silva, D. N., Oliveira Freitas, J. C. (2020). A novel oil-in-water microemulsion as a cementation flushing fluid for removing non-aqueous filter cake. Journal of Petroleum Science and Engineering, 184, 106536. doi: http://doi.org/ 10.1016/j.petrol.2019.106536

Denis Maryanov, Postgraduate Student, Department of Vessel Power Plants, National University Odessa Maritime Academy, Odessa, Ukraine, e-mail: denismaryanovv@gmail.com,ORCID: https://orcid.org/ 0000-0002-1355-5844 